\title{
Research on the Construction Path of Intelligent Financial Sharing Platform Under the Background of Industrial Interconnection
}

\author{
Guangmin $\mathrm{Fu}$ \\ Accounting School of Changchun University of Finance and Economics, Changchun, China \\ *Corresponding author. Email: 794398108@qq.com
}

\begin{abstract}
In 2018, the scale of China's digital economy has reached up to 31.3 trillion, accounting for $4.8 \%$ of China's GDP. In the fourteenth five-year plan, the concept of "Digital China" was clearly put forward again. And the digital economy has been developing rapidly. Starting from the background of industrial interconnection, relying on the preconditions of big data center construction project in Jilin Province, comparing with the development status and trends at home and abroad, this paper combs out the existing problems of traditional financial process in data collection, specification, utility and use, and proposes to start from the integration of business and financial process, build standardized sharing process, and finally, with the help of OCR, RPA and AIIt also analyze the specific application scenarios of the platform in different fields of universities, large enterprises and institutions, financial accounting and consulting enterprises and the government.
\end{abstract}

Keywords: Financial sharing platform, Intelligent, Sharing.

\section{INTRODUCTION}

According to the statistical report on Internet development in China released by China Internet Network Information Center, the Internet penetration rate in China is $61.2 \%$ by the first half of 2019. Finance from computerization, cloud to financial intelligence, the traditional financial model has been broken, as the cornerstone of the new financial management world, intelligent finance, as the logo of the new era, is becoming more and more clear. With the gradual maturity of the application of big data, cloud platform, AI and other technologies in Jilin Province, the establishment of Intelligent Financial Sharing Service Center has become an inevitable trend of enterprise development.

"China industry digital report 2020" points out that co construction, sharing and symbiosis have become the key to industrial digital transformation. Therefore, in the era of symbiosis, enterprises need to open their boundaries, connect and share. In order to better realize the strategic transformation of financial digitalization, they need to create an exclusive digital ecosystem. Enterprises need to form a community of common destiny with employees, partners and customers, promote financial development, broaden their horizons, give full play to their financial capabilities, establish deeply integrated standardized data, and provide realtime intelligent decision-making And analysis, to build the core competitiveness.

Based on the premise of Jilin big data center construction project, combined with the theory and practice of process optimization, this paper builds a standardized, controllable and end-to-end intelligent financial sharing platform to upgrade the traditional financial work, so as to improve the quality of accounting information, improve the efficiency of accounting work and reduce the cost of accounting work.

\section{THE DEVELOPMENT STATUS AND SIGNIFICANCE OF INTELLIGENT FINANCIAL SHARING PLATFORM AT HOME AND ABROAD}

\subsection{Development Status at Home and Abroad}

Every year, Gartner will release the top ten strategic technology development trends for the next year. There are two standards for these strategic technologies: first, 
some disruptive technologies that will have a profound impact on all industries, all enterprises and human life; second, these technologies may not be fully mature yet. The top ten strategic technologies in 2019 involve three themes of intelligence, digitization and grid, and the top ten strategic technologies in 2020 involve two themes of people-centered and intelligent space. It can be seen that intelligent technology will have a profound impact on all industries and enterprises, and the financial field is no exception. In the era of digital economy with surging new technologies such as "great wisdom moving cloud area", data has become a new factor of production (CPC Central Committee and State Council, 2020), and "using data to endow wisdom in the cloud" has become a new driving force for leading economic development (National Development and Reform Commission and central network information office, 2020).In this context, intelligent finance has become a new thing faced by both academic and practical circles, and its theoretical and practical exploration has become a hot spot in recent two years, which has aroused widespread social concern.

In the field of theoretical research, the 40 year development of accounting informatization in China has experienced three stages: accounting computerization, accounting informatization (narrow sense) and accounting intelligence (Liu Qin and Yang Yin, 2019).Intellectualization and standardization have become the transformation direction of Financial Sharing Service Center (Chen Hu et al., 2017; Li Wenyi et al., 2019; Jia Xiaoqiang et al., 2020). Sharing 4.0 is the intelligent service center, which aims to quickly accumulate big data for enterprise operation, and realize data empowerment, decision support and intelligent service (Liu Dantong, 2018). The intelligent financial platform can be divided into three levels: accounting type, management type and strategic type (Liu Qin and Yang Yin, 2018).In the field of financial practice, the discussion on the construction of intelligent finance is still in a state of dot: some typical intelligent application scenarios in the financial field have been realized, such as intelligent reimbursement, intelligent accounting and intelligent operation services (Tang Yong, 2018), as well as automatic collection and payment (Liu Dantong, 2018), automatic tax declaration, etc; Some possible application directions of intelligent finance are proposed, such as financial forecasting, operation deduction, risk quantification, value optimization, decision automation and information recommendation (Han Xiangdong and Yu Hongyan, 2018);Some preliminary intelligent financial systems began to appear, such as the artificial intelligence accounting system (tarmidi et al., 2018) for small and mediumsized enterprises to solve the problem of intelligent document recognition, and the intelligent reimbursement platform generally provided by domestic manufacturers.

\subsection{Development Trend}

Literature review and practice show that intelligent finance is a new thing in the field of Finance in recent two years. The top management of relevant departments attaches great importance to the application of new technologies such as blockchain and artificial intelligence in the field of accounting informatization, which is one of the three important tasks that the Ministry of finance will focus on in the next step in the field of accounting informatization. However, the current research only focuses on the concept and thinking of intelligent finance to carry out a preliminary discussion. There are some tentative explorations in the field of practice and some results have been achieved. There are few systematic discussions on the construction of enterprise intelligent finance. Based on the opportunity of "new infrastructure construction" in Jilin Province, this paper systematically explains and discusses the basic framework and construction ideas of building intelligent financial sharing platform. Starting from the background and current situation of intelligent financial research and practice, this paper discusses the definition, characteristics and basic framework of Intelligent Financial Sharing at the theoretical level, and discusses the construction of Intelligent Financial Sharing Platform with an enterprise as an example at the application level The overall thinking and construction path of the project.

\subsection{Theoretical and Practical Significance}

The importance of Finance in enterprise management is self-evident, and financial data itself is an important indicator and decision-making basis to measure and evaluate enterprise operation performance. With the opportunity of "new infrastructure construction" in Jilin Province, under the background of digital intelligence sharing, this paper discusses the definition, characteristics and mechanism of digital intelligence Financial Sharing from the theoretical level, systematically explains and discusses the basic framework and construction ideas of financial enabling enterprise value creation, redefines the understanding of finance as an information system and decision-making useful theory, and broadens the existing perspective of financial research.

The top management of relevant departments attaches great importance to the application of big data, artificial intelligence and other new technologies in the field of accounting informatization, which is one of the three important tasks that the Ministry of finance will focus on in the next step in the field of accounting informatization. However, the current research only focuses on the concept and thinking of intelligent finance, and there are some tentative explorations in the field of practice, and some results have been achieved. 
With the help of the research results of this topic, it has a good application value in improving the efficiency of the relevant government departments, the value creation ability of enterprises and institutions, and the talent training of university scientific research institutes.

\section{SORT OUT THE MAIN PROBLEMS EXISTING IN THE TRADITIONAL FINANCIAL PROCESS}

Data collection: the same type of data collection requires a lot of time and manpower. For example: every day, financial personnel download bank slip with different formats from different banking systems, and match a / $\mathrm{R}$ and a / $\mathrm{P}$ records according to certain rules. Except for a small part that can complete the matching, most of the rest need to communicate with the front desk by phone, e-mail or even to the store for several rounds.

Data specification: Although the traditional financial accounting has standards and standards to follow, data collection is scattered in different places, and enterprises will selectively implement the standards according to their own needs. This directly leads to the data range is not unified, accounting caliber is not unified and other issues, is not conducive to horizontal comparison between enterprises, management is difficult to make correct decisions.

Data utility: the traditional financial department's transactional work (such as auditing vouchers, bookkeeping, reconciliation and statements, etc.) takes up a lot of time, which leads to the failure of the functions that finance should perform, such as supporting the company's strategy, paying attention to changes in the external environment and participating in performance appraisal; the traditional financial accounting system determines the single afterwards orientation of financial work, that is, to wait until after the business occurs Making account books and preparing financial statements lead to the fact that finance can not effectively participate in the process of business management.

Data use: in the account book setting and the use of subjects, the traditional financial personnel did not set and use in strict accordance with the accounting standards and relevant financial systems, and the financial accounting lacked controllability and accuracy, which led to the confusion of accounts, accounts, account tables and other serious inconsistencies, which led to the distortion of financial information and the dislocation of enterprise strategy.

\section{CONSTRUCTION PATH OF INTELLIGENT FINANCIAL SHARING PLATFORM}

In this paper, with the help of the following methods, combined with theory and practice, scientific and reasonable to build intelligent financial sharing platform. First, through literature review to obtain the theoretical and practical information related to the project research, to provide a scientific theoretical basis for the project; second, research interview method. To investigate the typical successful cases of intelligent financial sharing platform, to do field investigation, to master the first-hand information, is more conducive to the implementation of the results. Using expert interview method, learning from the opinions of relevant professional experts, combined with the opinions of experts in the practical field, to enhance the application value of the research results of this topic; third, process optimization method. In the era of industrial interconnection, the front-end business process is gradually online, so we need to consider using scientific and technological means to match the financial process online, and realize the optimal allocation of financial process. Therefore, in order to achieve the balance between efficiency and quality in the process of optimizing the financial process, this project mainly adopts the process optimization method of considering efficiency (Standardization) and quality (personalization).

First, integrate business and financial processes. After the establishment of the financial sharing platform, it has opened up the information island, and realized the interconnection of shared finance, business finance and Strategic Finance. On the one hand, through financial support business, to ensure that the strategy and planning formulated by the strategic financial department can be implemented in the enterprise; on the other hand, through sharing the data provided by finance and the actual operation of the business department, the use of management accounting tools to generate effective information for strategic financial department reference, improve management efficiency and create value for the enterprise. This part is mainly based on the intelligent means (using OCR + RPA + AI, etc.) to first classify the business, and then match the standardized financial process to implement the intelligent integration of industry and finance.

Secondly, build standardized sharing process. The root of solving the sharing problem lies in the "Sharing Construction of financial service process", and the realization of sharing first needs process standardization. Specific implementation: first classify the business, and then determine the corresponding system (i.e. standardized transformation, unified coding, employee coding, Department coding, customer coding, supplier coding, entry project coding, etc.), and then 
corresponding to the financial process, finally realize the "end-to-end" standardized process.

Finally, the key to realize the final landing of the intelligent financial sharing platform is to use OCR, RPA, AI and other scientific and technological intelligent tools and technologies to automatically, quickly, accurately and continuously process financial accounting and management, help financial personnel to release the energy of routine work, and work more in need of insight, negotiation ability and creative thinking, so as to achieve the goal of using the intelligent platform Data, control the whole operation process.

At the same time, the final realization of intelligent financial sharing needs the seamless docking of the front (business), middle (Finance) and back (Technology) platforms. In addition to the application of advanced technology, it also needs the consciousness of all departments to be consistent and cooperate together to help the landing of the platform.

\section{APPLICATION SCENARIOS OF INTELLIGENT FINANCIAL SHARING PLATFORM}

Colleges and universities. The project team will think about the relevant theory and practice of intelligent finance, and transform it into the curriculum system of accounting talents training, which is conducive to the cultivation of financial talents suitable for social development in Colleges and universities, and promote the implementation of industry university research projects.

Large enterprises and institutions. They can build their own financial sharing platform, which is conducive to the centralized management of enterprise groups and the formulation of strategies. It solves the problem of repeated information collection and effectively reduces the labor cost. Because the data of the current system can not be shared with the industry, it inevitably leads to the repeated collection of the same information, which not only increases the burden of work, but also destroys the consistency of the data. Building an intelligent financial sharing platform will effectively alleviate the above problems, realize the integration of industry and finance and information resource sharing, improve work efficiency and save labor costs.

Financial accounting and consulting enterprises. For small and medium-sized enterprises which are short of funds, it is not realistic to spend a lot of capital to build a financial sharing platform (enterprises focus on the improvement of core business level). At present, more than $50 \%$ of SMEs need to outsource their financial accounting work. Building an intelligent financial sharing platform can not only save the operating costs of small and medium-sized enterprises, but also stimulate market vitality.
The government. The construction of intelligent financial sharing platform can greatly improve the supervision level of relevant government departments, effectively improve the work efficiency of law enforcement personnel, and alleviate the contradiction between heavy workload and insufficient staff. It is conducive to improving the level of internal and external resource allocation of the government.

\section{CONCLUSIONS}

The construction of financial intelligence and sharing can not be achieved overnight. On a basis of their own business characteristics, enterprises need to formulate perfect implementation strategies, overall planning and step-by-step implementation. Financial personnel need to develop a systematic intelligent financial innovative thinking, ensure the smooth function of concept, personnel, process and system, and ultimately reduce costs and increase efficiency for the enterprise and improve governance.

\section{ACKNOWLEDGMENTS}

Jilin Provincial Department of education project "Research on the construction path of Intelligent Financial Sharing Platform under the background of industrial interconnection" (Project No.: JJKH20211394SK)

\section{REFERENCES}

[1] Pan Nan. Research on intelligent automation business process optimization of ABC Group Financial Sharing Service Center [D]. Southeast University, 2019

[2] Zhang min. ten hot issues of intelligent finance [J]. Monthly journal of accounting, 2021 (02): 25-30

[3] Yu Yifan. Construction of cloud platform for Financial Sharing Service Center in the era of big data $[\mathrm{J}]$. Accounting of township enterprises in China, 2021 (02): 139-140

[4] Wang Dan. Discussion on the construction of Financial Sharing Service Center in the era of big data [J]. Public investment guide, 2020 (19): 58-59

[5] Liu Xiaonan. Research on the construction of Financial Sharing Service Center under the background of big data era $[\mathrm{J}]$. China management informatization, 2020,23 (17): 89-91

[6] Kou Jianwen. Strategy for the establishment of Financial Sharing Service Center of enterprise group in the era of big data $[\mathrm{J}]$. Enterprise reform and management, 2019 (23): 133-134

[7] Jilin Provincial People's government. Notice of Jilin Provincial People's Government on printing and distributing the implementation plan of new infrastructure "761" project in Jilin Province, 2020(4) 